\title{
A SCANNING ELECTRON MICROSCOPIC STUDY OF THE PATTERNS OF EXTERNAL ROOT RESORPTION UNDER DIFFERENT CONDITIONS
}

\author{
Ravindran SREEJA ${ }^{1}$, Chaudhary MINAL ${ }^{2}$, Tumsare MADHURI ${ }^{3}$, Patil SWATI ${ }^{4}$, Wadhwan VIJAY ${ }^{5}$
}

\author{
1- B.D.S, III year postgraduate, Department of Oral Pathology and Microbiology, Sharad Pawar Dental College, Maharashtra, India. \\ 2- M.D.S (Oral and Maxillofacial Pathology and Microbiology), M.D.S (Orthodontics and Dentofacial Orthopedics), Professor and Head of Department, \\ Department of Oral Pathology and Microbiology, Sharad Pawar Dental College, Maharashtra, India. \\ 3- M.D.S (Oral and Maxillofacial Pathology and Microbiology), Professor, Department of Oral Pathology and Microbiology, Sharad Pawar Dental \\ College, Maharashtra, India. \\ 4- M.D.S (Oral and Maxillofacial Pathology and Microbiology), Assistant Professor, Department of Oral Pathology and Microbiology, Sharad Pawar \\ Dental College, Maharashtra, India. \\ 5- M.D.S (Oral and Maxillofacial Pathology and Microbiology), Reader, Department of Oral Pathology and Microbiology, Sharad Pawar Dental \\ College, Maharashtra, India.
}

Corresponding address: Dr. RAVINDRAN. SREEJA - III rd year P.G Student - Department of Oral Pathology and Microbiology - Sharad Pawar Dental College - Sawangi (Meghe) - Wardha-442004 - Maharashtra - India - Phone: 09960811170 - e-mail: sri adorned@rediffmail.com

Received: July 9, 2008 - Modification: August 31, 2008 - Accepted: October 15, 2008

\begin{abstract}
$O$

bjective: The aim of this study was to examine if there are qualitative differences in the appearance of external root resorption patterns of primary teeth undergoing physiologic resorption and permanent teeth undergoing pathological root resorption in different conditions. Material and Methods: A total of 40 teeth undergoing external root resorption in different conditions were divided into 4 groups and prepared for examination under scanning electron microscopy at magnifications ranging from 20x to 1000x. Group I: 10 primary molars exfoliated due to physiologic root resorption; Group II: 10 permanent teeth with periapical granulomas showing signs of resorption; Group III:10 permanent teeth therapeutically extracted during the course of orthodontic therapy with evidence of resorption, and Group IV: 10 permanent teeth associated with odontogenic tumors that showed evidence of resorption. Results: In Group I, the primary teeth undergoing resorption showed smooth extensive and predominantly regular areas reflecting the slow ongoing physiologic process. In Group II, the teeth with periapical granulomas showed the resorption was localized to apex with a funnel shaped appearance in most cases. Teeth in Group III, which had been subjected to a short period of light orthodontic force, showed the presence of numerous resorption craters with adjoining areas of cemental repair in some cases. Teeth associated with odontogenic tumors in Group IV showed many variations in the patterns of resorption with extensive loss of root length and a sharp cut appearance of the root in most cases. Conclusion: Differences were observed in the patterns of external root resorption among the studied groups of primary and permanent teeth under physiologic and pathological conditions.
\end{abstract}

Key words: Root resorption. Scanning electron microscopy.

\section{INTRODUCTION}

The hard tissue of human teeth is composed of enamel, cementum and dentin. Multinucleated cells called odontoclasts resorb root dental hard tissues ${ }^{9}$.The resorption of hard tissue in primary teeth is a normal physiologic phenomenon that leads to their exfoliation and ultimately the transition to permanent dentition.

The hard tissues of permanent teeth, on the other hand, are not resorbed under normal conditions and resorption is a pathologic process. This pathologic root resorption process has been broadly classified based on the site of occurrence into external or internal resorption ${ }^{2}$. Several causes have been attributed to external resorption including inflammation, pressure, and certain systemic disorders.

Various methods have been advocated to assess resorption including histology ${ }^{7}$, the use of panoramic and periapical radiography ${ }^{6}$ and scanning electron microscopy $(\mathrm{SEM})^{3}$. The use of traditional methods, such as histologic and radiographic analyses to study this process has inherent shortcomings. The use of SEM has certain advantages over the other traditional methods, as it permits the assessment in multiple planes. The purpose of exploring morphology is to get an insight into function. The morphologic image represents the visual expression of functional activity and thus the aim of this study was to examine if there are 
qualitative differences in the appearance of external root resorption patterns of primary teeth undergoing physiologic resorption and permanent teeth undergoing pathological root resorption in different conditions.

\section{MATERIAL AND METHODS}

\section{Sample Collection}

A total of 40 human teeth were collected over a period of 3 months and divided into 4 groups. All teeth were included in the study after being examined under a stereomicroscope at 10x magnification for the presence of root resorption areas. Group I:10 primary molars that exfoliated due to physiologic root resorption; Group II: 10 permanent teeth associated with periapical granulomas showing signs of pathologic resorption due to the inflammatory process involved; Group III: 10 premolars subjected to a short duration of light orthodontic force by conventional mechanics for a period of less than 2 months and therapeutically extracted during the course of therapy; Group IV: 10 permanent teeth associated with odontogenic tumors.

\section{Sample Preparation}

After extraction and removal of the soft tissue remnants, all teeth were immediately immersed in 5\% sodium hypochlorite solution to remove the remaining nonmineralized organic components. The roots were separated from the crown and then dried in a desiccator for $24 \mathrm{~h}$ prior to analysis. The roots were then mounted on the stubs using double-sided adhesive tape and sputter coated with platinum before examination. The specimens were then analyzed in a scanning electron microscope (JEOL JSM 6380A, JEOL, Tokyo Japan) at $20 \mathrm{kv}$ beam current. The specific areas of interest on the teeth were examined under various $20 \mathrm{x}, 100 \mathrm{x}$, $250 \mathrm{x}, 500 \mathrm{x}$ and $1000 \mathrm{x}$ magnifications. The lowest magnifications were used to identify the areas of resorption and the higher magnifications to study the specific patterns of resorption. Areas of the roots undergoing resorption were compared to that of a normal root and presence of any recurring pattern was noted. The resorption patterns were described on the basis of extent and the distinct surface appearance.

\section{RESULTS}

\section{Group 1}

Figure $1 \mathrm{~A}$ is the image of a primary molar undergoing resorption examined at $12 \mathrm{x}$ magnification. Presence of extensive areas of resorption can be noticed on the lingual aspect of all roots leading to blunting of the apical regions. Extensive loss of cementum from the entire lingual aspect of the roots is visible. The extent of resorption of the different roots was not however uniform. The shaded area in Figure $1 \mathrm{~A}$ was selected for higher magnification and assessment. Figure $1 \mathrm{~B}$ is a higher magnification $(100 \mathrm{x})$ of the apical region that reveals rounding and blunting of this region. The shiny white area inside the apical foramen (dark arrow) is nothing but remnant of pulpal soft tissue. There is a loss of normal smooth topography of the root surface that now shows the uniform presence of numerous irregularly shaped concavities. The resorption area has a smooth multilocular appearance. In Figure 1C (at 500x), the presence of several individual resorption lacunae can be clearly appreciated. The area looks as if individual adjacent areas of tooth material have been scooped out (dark arrows) from the root surface. In Figure 1D (1000x), the dentinal tubule entrance is visible (dark arrow).

\section{Group 2}

Figure $2 \mathrm{~A}$ shows a mandibular permanent molar undergoing root resorption due to an associated periapical granuloma .There is a blunting of the entire apical region of the roots that is evident at $10 \mathrm{x}$ magnification. At this magnification the entire apical root surface has a smooth eggshell like appearance. At the apical region, however, there is also evidence of resorption occurring near the foramen (dark arrow). This region was chosen for closer examination. In Figure 2B (100x) there is clear evidence of resorptive activity seen around the periapical region (dark arrow). Areas of resorption can also be appreciated in the apical third of the root, particularly close to the apex, leading to a funnellike appearance. There is a widening of the apical foramen diameter due to the loss of cementum around the periapical region. The resorptive activity seems to be radiating outward from the apex. There is also the presence of a clear border demarcating the affected and unaffected areas at the apical foramen region (light arrow). In Figure 2C (250x), the periphery of the foramen shows areas of normal cementum interspersed with resorption craters (dark arrows and light arrows). Differently from the primary teeth, the resorptive areas are not uniformly seen. In Figure 2D (500x), the individual craters of resorption are more visible (dark arrow).

\section{Group 3}

In Figure 3A, the image shows a mandibular first premolar undergoing pressure resorption during the course of orthodontic treatment resorption seen mostly along mesial and buccal aspect. Active resorption is present even though the tooth was subjected to orthodontic force for a relatively short duration of less than two months. Here areas of resorption are (dark arrows) seen along most of the entire surface leading to loss of normal smooth appearance of this surface. There was no evidence of any resorptive activity on the opposing aspect. A slight tilt of the specimen was carried out so that a more detailed analysis of the mesial aspect could be undertaken. In Figure 3B (100 x), the presence of resorption bays (dark arrows) with adjoining areas of cemental repair (light arrow) can be appreciated. In Figures 3C and 3D (250 and 500x), the presence of an individual resorption crater of irregular size and shape is specifically visible (dark arrow) with adjoining normal cementum (light arrow). 


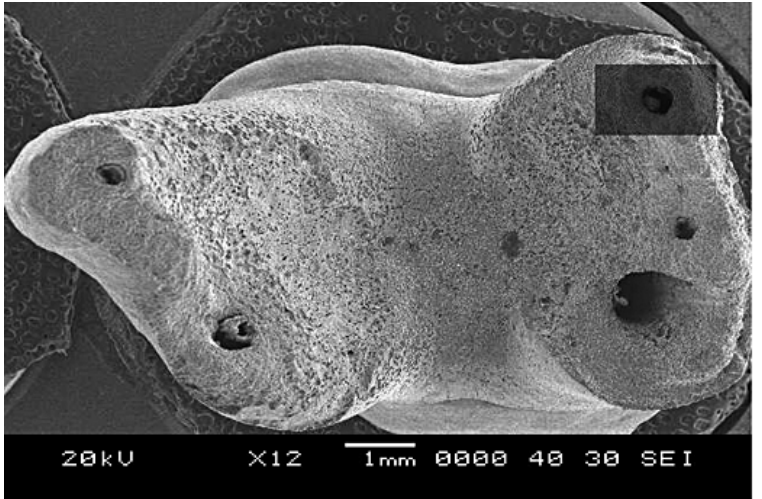

A

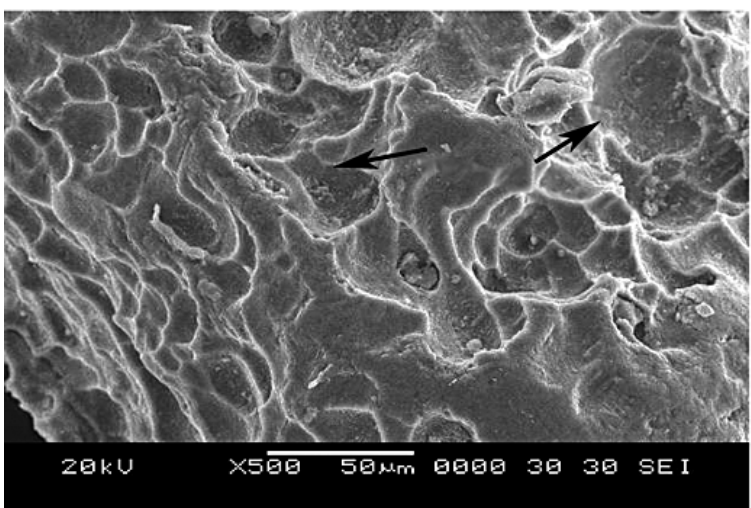

C
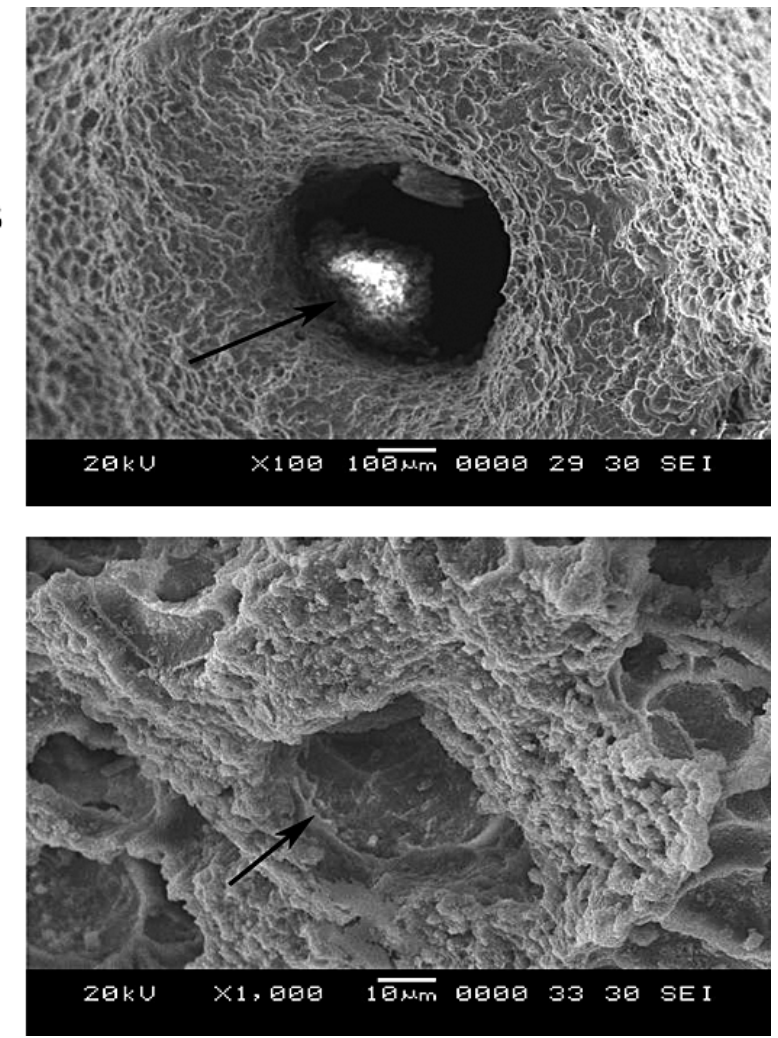

FIGURE 1 (A-D)- Root resorption of a primary molar at 12x, 100x, 500x and 1000x magnifications

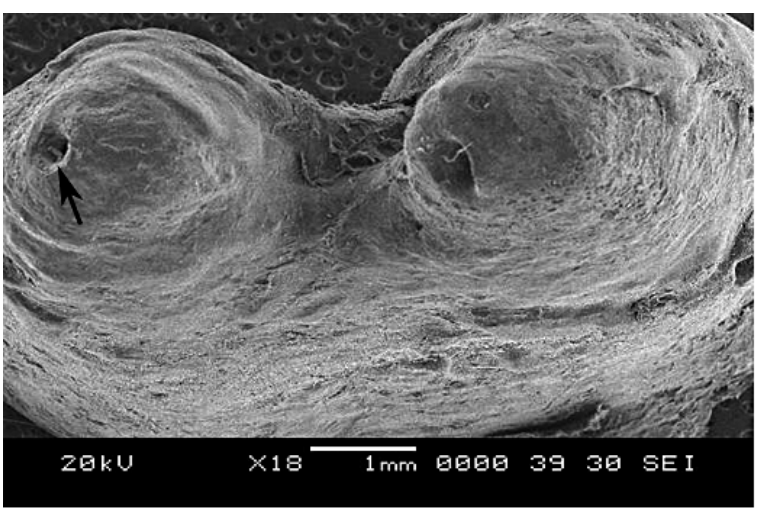

A

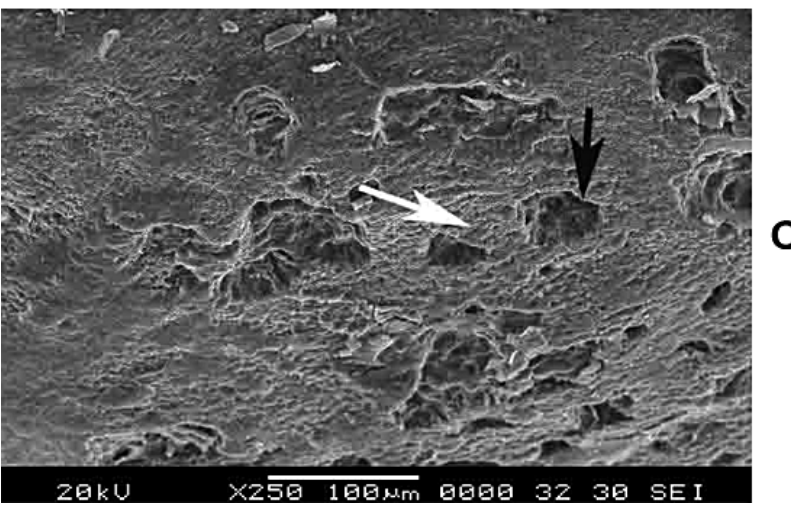

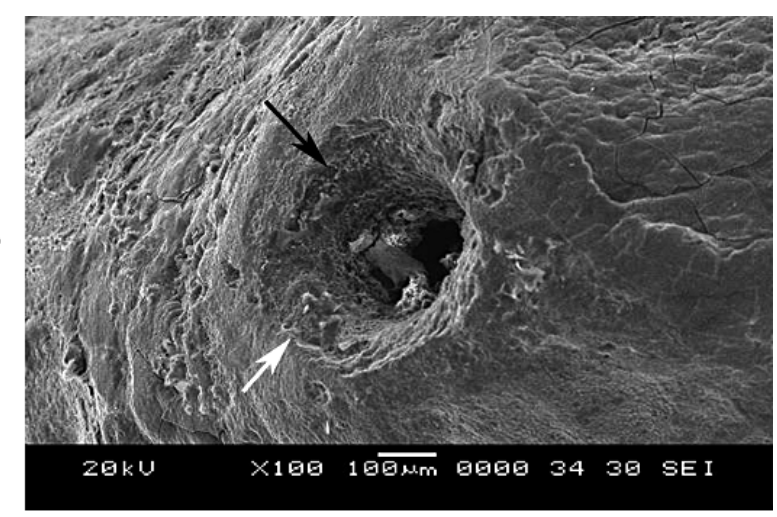

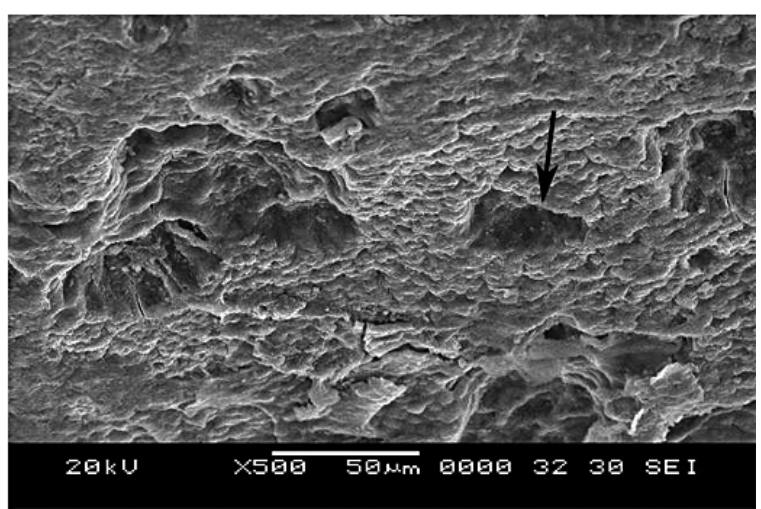

FIGURE 2 (A-D)- Root resorption of permanent molar associated with periapical granuloma at 18x, 100x, 250x, 500x magnifications 


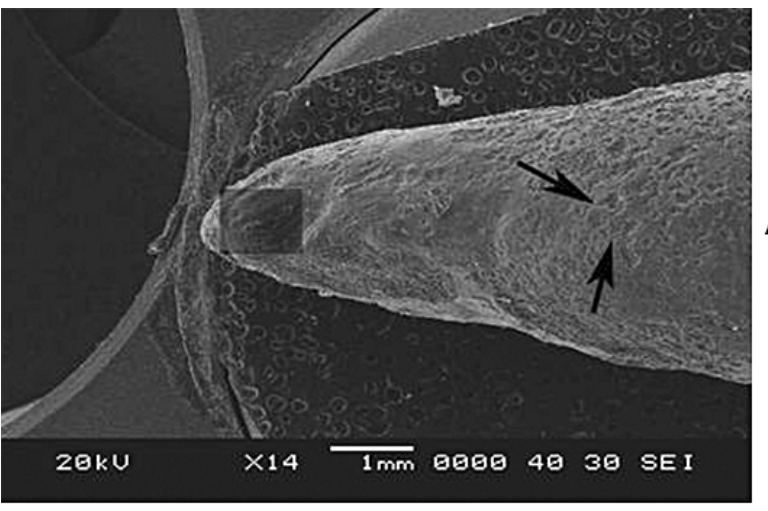

A

B
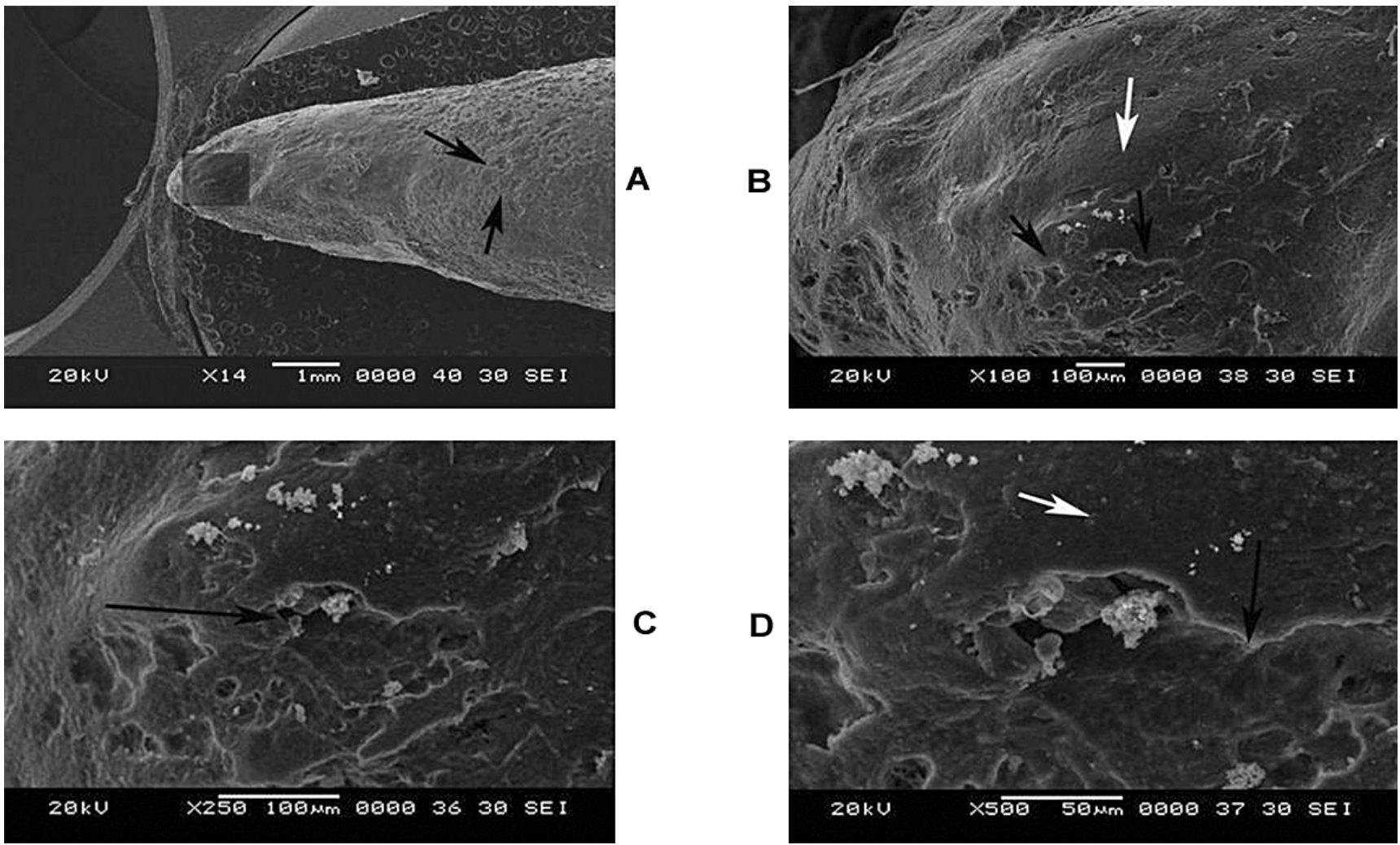

C

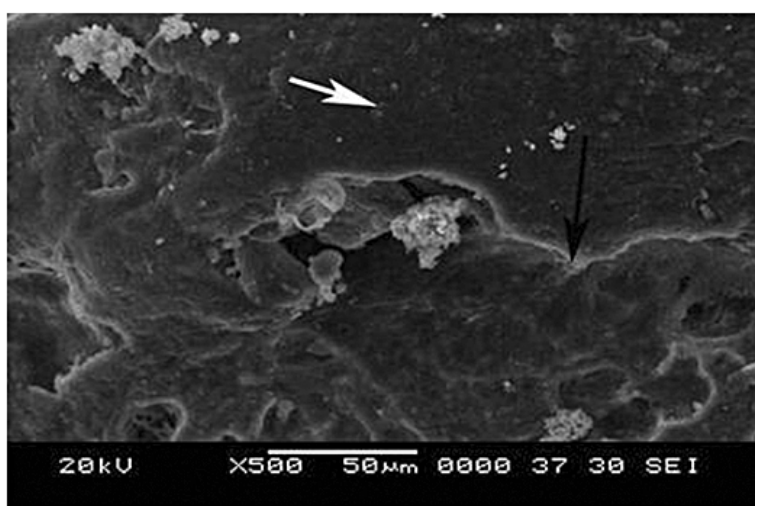

FIGURE 3 (A-D)- Root resorption of a premolar subjected to orthodontic force at 14x, 100x, 250x, 500 magnifications

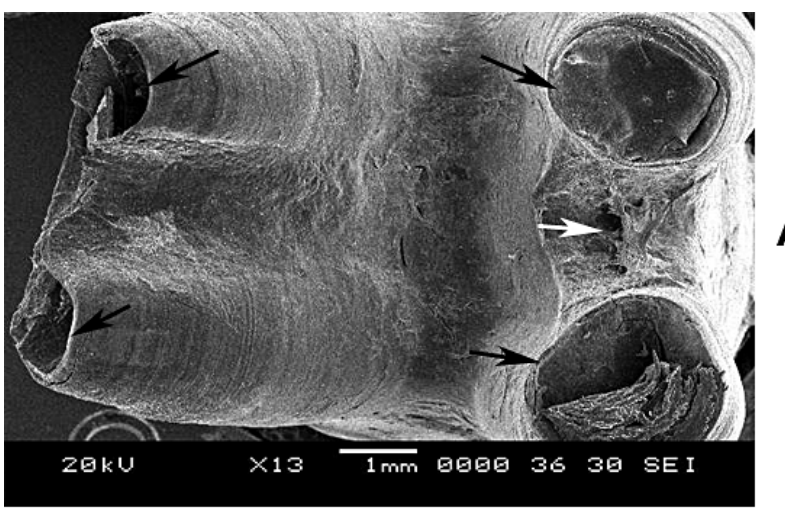

A
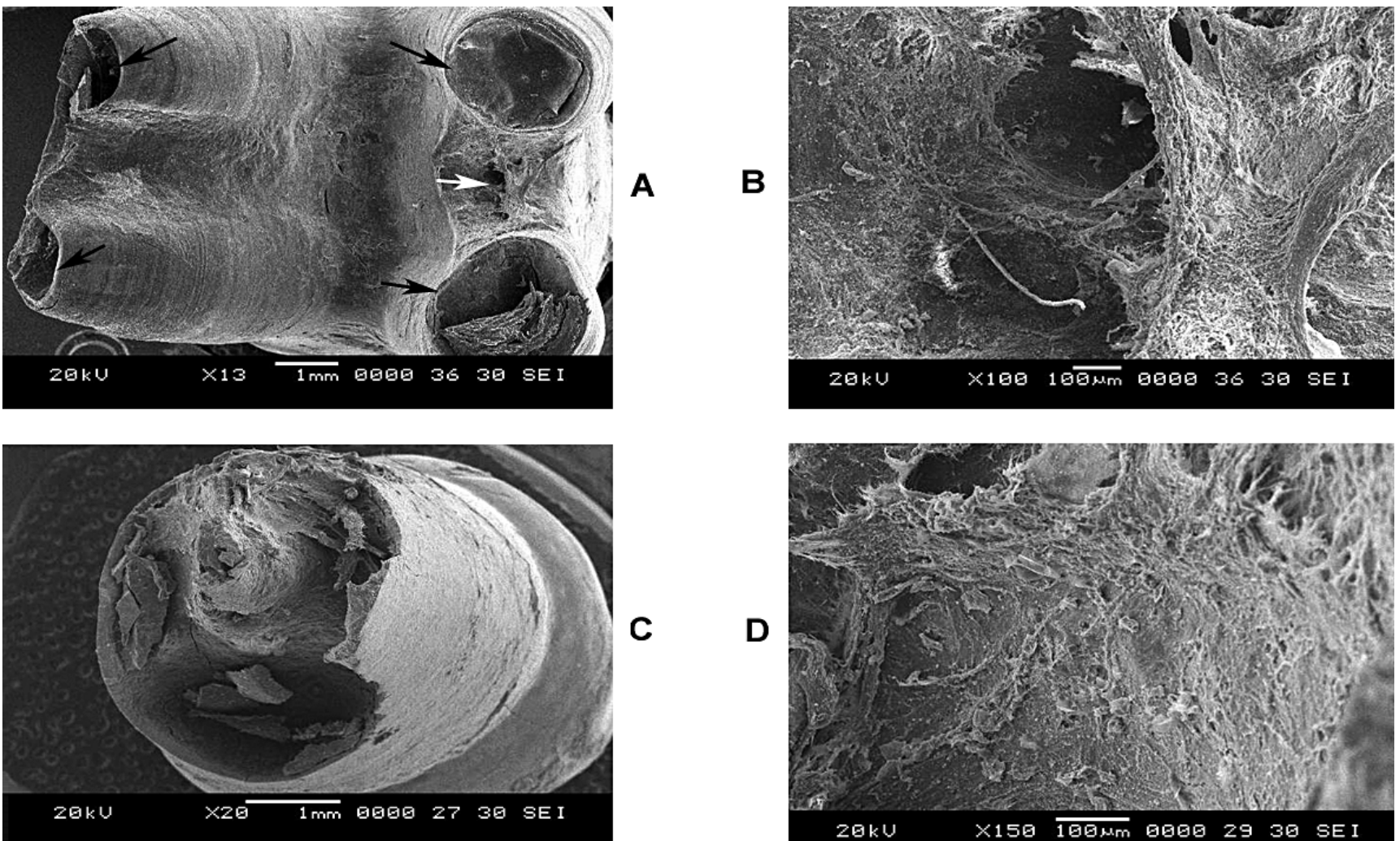

C

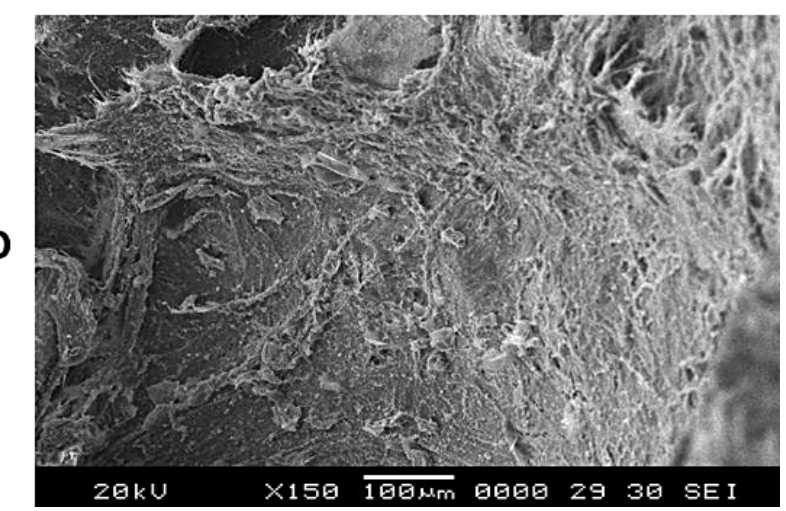

FIGURE 4 (A-D)- Root resorption patterns of a permanent molar (13 and 100x) and a permanent incisor (20 and 150x) associated with odontogenic tumor 


\section{Group 4}

In Figure 4A (13x), which shows a permanent molar associated with an ameloblastoma, the most striking feature is the extensive loss of root length as a result of the resorption. Resorptive activity is evident in each of the individual roots and even in between them (light arrows) with differing appearance. However interestingly unlike the smooth blunting of the apex seen in the earlier conditions the roots have a sharp cut appearance (dark arrows). In Figure 4B (70x), the presence of a single very large resorption crater from in between the roots is seen (dark arrow). Higher magnifications (250 and 500x) did not reveal any specific patterns of resorption but loss of normal smooth root surface topography. Figure 4C (20x) shows a haphazard and ragged appearance of the root of an anterior tooth with extensive loss of root length. Figure 4D (150 x) reveals loss of normal smooth root surface topography.

\section{DISCUSSION}

Different causes have been attributed to the root resorption process including pressure, inflammation, neoplastic process and systemic conditions. The different causes of resorption in physiological and pathological conditions may lead to a diverse resorptive potential by the odontoclasts. As the morphologic image represents functional activity various patterns of resorption can be expected in different conditions. It was our intention to look at resorption patterns resulting from different pathological and physiological processes applying SEM. The use of histologic sections or radiographs for studying root resorption may lead to missing areas of resorption in certain planes of interest or even small areas of transient resorption may not show up in these methods. SEM on the other hand permits visualization of surface structures and is particularly valuable in recording three-dimensional morphology of mineralized tissues. This technique provides enhanced visual assessment of root surfaces, unattainable with histological models reconstructed from serial sections. The ability to tilt the mounted sample in all three planes of space to a certain degree while analyzing it permits us to focus on specific areas of interest.

In the present study, the primary teeth examined with SEM were in different stages of root resorption but most of them had lost more than two thirds to three quarters of their root length prior to exfoliation. To this day it is believed that the emerging permanent tooth applies pressure on the primary predecessor leading to activation of odontoclasts and ultimately exfoliation ${ }^{4}$. Though the resorption of primary teeth is not solely dependent on the pressure of erupting permanent teeth the site of resorption in all the primary molars studied was in close proximity to the succeeding permanent follicle. Another notable feature was that the resorption was of varying extent in different roots of the same molar. Roots closer to the follicle showed a greater degree of resorption with extent of root divergence and inter root distant affecting the final outcome. It has been reported that physiologic process is a not a continuous process but an intermittent process with periods of resorption alternating repair activity. However, in all the primary molars examined at various magnifications in the present study the pattern of root resorption in primary teeth had a regular appearance. This pattern is probably a reflection of the slow ongoing physiologic process of root resorption.

On the other hand, the site of the lesion in periapical granuloma accounts for the severity and localization of the resorptive process to the vicinity of the apical region. The funnel-like appearance seen in our study has previously been described $^{5}$. This indicates that resorption is actively taking place mainly along the localized areas of pressure from the periapical granuloma. The areas of resorption were localized at the apex with a clear border demarcating it from the adjoining normal area indicating that pressure from the lesion could be a major contributing factor to the process.

Orthodontic tooth movement is possible as cementum has greater resistance to resorption than bone. However, root resorption is also seen after the application of light orthodontic force even within a short-duration period ${ }^{10}$. The point of force application with conventional orthodontic mechanics is from the buccal aspect. In the early stages of treatment, this causes crown buccal and root lingual tipping of premolars leading to opposing areas of tension and compression in the crown and root. The resorption process on examination with SEM was however not wholly restricted to any particular area. Based on extent varying degrees of orthodontically induced inflammatory root resorption have been described ${ }^{1}$. In the present study, the resorption in all premolars was restricted to mild surface cemental resorption probably because of the short duration for which teeth were subjected to force application. The extent and site of resorption was not uniform to reach a categorical conclusion as a multitude of local and systemic factors play a vital role in orthodontically induced root resorption. It is important to know that even in early stages of orthodontic treatment there is evidence of cemental resorption, which may not always be evident on radiographs unless it reaches an advanced stage.

In the teeth associated with odontogenic tumors, no particular pattern was discernable as there were variable in appearance. Most teeth, however, showed an extensive loss of root length form the resorptive process. Previously there have been reports of root resorption in teeth in the proximity of odontogenic tumors, such as ameloblastoma ${ }^{8}$. Two teeth associated with ameloblastomas were chosen for closer examination as they had a strikingly different appearance of roots. A sharp cut appearance of the roots was visible echoing the aggressive nature of the rapidly growing lesion. Root resorption was haphazard and ragged as previously described in literature. This aggressive root resorption pattern reflects the loss of cellular regulatory control synonymous with tumor pathology. 


\section{CONCLUSIONS}

This is an interesting research study for the knowledge of the root resorption pattern under different physiological and pathological conditions. There were differences in the patterns of external root resorption that resulted from different conditions when studied under SEM. In most cases, the resorption pattern reflected the nature of the underlying etiology. Although study is valuable as an additional source of basic information, it has limited clinical application. Further investigations on the quantitative and molecular aspects of root resorption will shed light on several unknown features providing a database for exciting new fields like forensic dentistry.

\section{ACKNOWLEDGEMENTS}

We would like to thank Dr. Pathak and the staff of the Department of Metallurgy at Visvesaraiyya National Institute of Technology, Nagpur, for their technical assistance and suggestions for carrying out the study.

\section{REFERENCES}

1- Brezniak N, Wasserstein A. Orthodontically induced inflammatory root resorption. Part I: the basic science aspects. Angle Orthod. 2002;72:75-9.

2- Gunraj MN. Dental root resorption. Oral Surg Oral Med Oral Pathol Oral Radiol Endod. 1999;88(6):647-53.

3- Han G, Huang S, Hoff JW, Zeng X, Kuijpers-Jagtman AM. Root resorption after orthodontic intrusion and extrusion: an intraindividual study. Angle Orthod. 2005;75(6):912-8.

4- Kronfeld R. The resorption of the roots of deciduous teeth. Dent Cosmos. 1932; 74:103-20.

5- Malueg LA, Wilcox LR, Johnson W. Examination of external apical root resorption with scanning electron microscopy. Oral Surg Oral Med Oral Pathol Oral Radiol Endod. 1996;82:89-93.

6- Sameshima GT, Asgarifar KO. Assessment of root resorption and root shape: periapical vs panoramic films. Angle Orthod. 2001;71:185-9.

7- Shiraishi C, Hara Y, Abe Y, Ukai T, Kato I. A histopathological study of the periodontal ligament tissue in root resorption in the rat. Arch Oral Biol. 2001;46:99-101.

8- Struthers P, Shear M. Root resorption by Ameloblastoma and cysts of the jaws. Int J Oral Surg. 1976;5(3):128-32.

9- Tencate AR, Nanci A. Physiologic tooth movement: eruption and shedding. In: Nanci A, editor. Tencate's oral histology: development, structure and function. St. Louis: Mosby; 2003. p. 275-98.

10- Weiland F. Constant versus dissipating forces in orthodontics: the effect on initial tooth movement and root resorption.Eur J Orthod. 2003;25:33542. 